\title{
Occultation by (22) Kalliope and its satellite Linus
}

\author{
M. Sôma ${ }^{1}$, T. Hayamizu ${ }^{2}$, K. Miyashita ${ }^{3}$, T. Setoguchi ${ }^{4}$ \\ and T. Hirose ${ }^{5}$ \\ ${ }^{1}$ National Astronomical Observatory of Japan, Mitaka, Tokyo 181-8588, Japan \\ email: mitsuru.soma@nao.ac.jp \\ ${ }^{2}$ Sendai Space Hall, Satsumasendai City, Kagoshima Pref. 895-0005, Japan \\ email: uchukan@bronze.ocn.ne.jp \\ ${ }^{3}$ Chikuhoku Junior High School, Omi Village, Higashi-Chikuma Gun, Nagano Pref. 399-7701, \\ Japan \\ email: k_miyash@js5.so-net.ne.jp \\ ${ }^{4}$ Japanese Occultation Information Network, c/o Kagoshima Hi-tech College, Kagoshima City, \\ Kagoshima Pref. 891-0141, Japan \\ email: set@bronze.ocn.ne.jp \\ ${ }^{5}$ International Occultation Timing Association, 1-13, Shimomaruko 1-chome, Ota Ku, Tokyo \\ 146-0092, Japan \\ email: thirose@cam.hi-ho.ne.jp
}

\begin{abstract}
The occultation of a 9.1 magnitude star by asteroid (22) Kalliope and its satellite Linus was successfully observed in Japan in 2006 November 7.826 UT. This was the first definite observation of an occultation of a satellite of an asteroid that was discovered previously by other means. As a result the position of the satellite relative to Kalliope was obtained to be $d=0.246 \pm 0.011$ (arcsec), and $P=313.8 \pm 2.7(\mathrm{deg})$, where $d$ is the angular distance and $P$ is the position angle. The derived size for Kalliope is $(209 \pm 40) \mathrm{km} \times(136 \pm 26) \mathrm{km}$ (with the major axis in position angle of $(8 \pm 17) \mathrm{deg})$, and that for Linus is $(33 \pm 3) \mathrm{km}$. From the observations, the occulted star is also found to be a close double star whose separation is about 0.7 mas in position angle of about $300 \mathrm{deg}$, and the magnitudes of the components are found to be almost the same $(\sim 9.9 \mathrm{mag})$.
\end{abstract}

Keywords. occultations, minor planets, asteroids, binaries: close

\section{Observations}

The occultation of the 9.1 magnitude star TYC 1886-01206-1 (SAO 78190) by asteroid (22) Kalliope and its satellite Linus was successfully observed in Japan at around 2006 November 7.826 UT. The occultation by Kalliope was predicted by the International Occultation Timing Association (IOTA) and the one by Linus was by Jerome Berthier, IMCCE (Institut de Mécanique Céleste et de Calcul des Éphémérides), France. Berthier's prediction was forwarded to us by Jean Lecacheux some 19 hours before the event. Those predictions were publicized to Japanese amateur observers through Japanese Occultation Information Network (JOIN), and observation results were also collected through JOIN.

The occultation by Kalliope was observed at eight stations and that by Linus was observed at other eight stations. Unfortunately the observations could not be made in the northern Japan due to bad weather. Five observers among the eight for Kalliope and three among eight for Linus used video equipment with precise time stamp for the observations so that their times were precise within about $0.03 \mathrm{sec}$. There was another observer for Linus who made the observation by video, but he failed to get precise times. 


\section{Results of the analysis}

From the observations the diameters of Kalliope and Linus, and the relative position of Linus to Kalliope at the time of the occultation were obtained by a weighted least squares method. The apparent figure of Kalliope is assumed to be an ellipse, and the lengths of its major and minor axes were obtained together with the position angle of the major axis, but for Linus only the western part was observed and furthermore its visual observations were not reliable (the event occurred by about one minute earlier than they expected because their observations were made according to the prediction of the occultation by Kalliope) and therefore for Linus we assumed the apparent figure to be a circle.

The analysis of the observations gives the following results:

the angular separation $d$ and position angle $P$ of Linus relative to Kalliope for the epoch 2006 November 7.826 UT of the occultation are:

$d=0.246 \pm 0.011(\operatorname{arcsec}) \quad P=313.8 \pm 2.7(\mathrm{deg})$.

The derived diameters for Kalliope are:

$(209 \pm 40) \mathrm{km} \times(136 \pm 26) \mathrm{km}$

and the position angle of the major axis is: $(8 \pm 17) \mathrm{deg}$.

The derived diameter of Linus is: $(33 \pm 3) \mathrm{km}$.

\section{Duplicity of the star}

Among the nine video observations eight video tapes of the observations were provided to us. While analyzing the video tapes we found that all of the videos indicated that the occulted star is a close double star. From the analyses of the light curves we have found that their separation is about 0.7 mas at a position angle of about $300 \mathrm{deg}$, and the magnitudes of the components are almost identical ( $~ 9.9 \mathrm{mag})$.

\section{Conclusion}

An occultation of a 9.1 magnitude star by (22) Kalliope and its satellite Linus was observed in total at sixteen stations in Japan at around 2006 November 7.826 UT. As a result the angular separation of $0.246 \pm 0.011$ (arcsec) of Linus relative to Kalliope in position angle of $313.8 \pm 2.7$ (deg) was obtained for the epoch of the occultation. The derived size for Kalliope is $(209 \pm 40) \mathrm{km} \times(136 \pm 26) \mathrm{km}$ (with the major axis in position angle of $(8 \pm 17) \mathrm{deg})$, and that for Linus is $(33 \pm 3) \mathrm{km}$. From the observation the occulted star is also found to be a close double star whose separation is about 0.7 mas at a position angle of about $300 \mathrm{deg}$, and the magnitudes of the components are found to be almost the same ( $\sim 9.9 \mathrm{mag})$. Details of the observations and analyses will be published elsewhere. 